\author{
Espacio y Desarrollo No 27, 2015, pp. 159-179 (ISSN 1016-9148) \\ http://dx.doi.org/10.18800/espacioydesarrollo.201501.008
}

\title{
BIOGEOGRAFÍA PREDICTIVA: TÉCNICAS DE MODELAMIENTO DE DISTRIBUCIÓN DE ESPECIES Y SU APLICACIÓN EN EL IMPACTO DEL CAMBIO CLIMÁTICO
}

\author{
Martín Timaná de la Flor, PhD \\ Pontificia Universidad Católica del Perú; Grupo Biogeociencias, \\ Departamento de Humanidades, Sección Geografía. INTE-PUCP. \\ mtimana@pucp.edu.pe \\ Lic. María Alejandra Cuentas Romero
}

Especialidad de Geografía y Medio Ambiente. Pontificia Universidad Católica del Perú;

Grupo Biogeociencias, INTE-PUCP. alejandra.cuentasr@pucp.edu.pe

\section{RESUMEN}

Se presenta el modelamiento de distribución de especies como una herramienta analítica para la biogeografía y para el estudio del impacto del cambio climático en la biodiversidad. Se revisan los conceptos y aplicaciones fundamentales de modelamiento; se describe el principio de máxima entropía así como su aplicación en el programa MaxEnt. Finalmente, se presentan ejemplos ilustrativos de modelamiento de dos especies vegetales con MaxEnt: Pycnophyllum spathulatum (Caryophyllaceae) y Prosopis pallida (Fabaceae), utilizando los datos climáticos disponibles en WorldClim tanto para las condiciones climáticas presentes como también bajo un escenario futuro de cambio climático (RCP 8.5) para determinar el impacto potencial de este en la distribución de las especies modeladas.

Palabras claves: modelamiento, análisis espacial, MaxEnt, cambio climático, biogeografía.

Predictive Biogeography: Species Distribution Modelling Techniques and Its Application to Climate Change Impact Assessment

\section{Abstract}

Species distribution modeling is presented as an analytical tool for biogeography and the study of climate change impact on biodiversity. Key modeling concepts and applications are reviewed; 
the maximum entropy principle is described as well as its implementation in the Maxent software. Lastly, two demonstrative examples of species modeling are presented using two plant species: Pycnophyllum spathulatum (Caryophyllaceae) and Prosopis pallida (Fabaceae). Bothe species were modeled using current climate data and under a future climatic change scenario based on RCP8.5 to examine the potential impact of global warming on the species' distribution.

Keywords: modeling, spatial analysis; MaxEnt, climate change, biogeography.

La biogeografía es considerada una rama de la geografía física cuyo objeto de estudio es la documentación y análisis de la distribución de especies y biotas sobre la Tierra. Basada en la información recopilada a lo largo de siglos en colecciones de historia natural, esta ciencia busca entender y explicar cómo y por qué los seres vivos se distribuyen en el espacio y el tiempo. Tal como lo indican Ehrlen y Morris (2015), la distribución de los organismos son la unidad fundamental del estudio biogeográfico. Como tal, constituye una herramienta fundamental para el manejo y conservación de la biodiversidad.

En las última décadas se ha observado, como resultado de una creciente actividad humana, una degradación acelerada de los ecosistemas naturales y extinción de especies (Millennium Ecosystem Assessment, 2005; Pimm et al., 2014). La deforestación, actividades extractivas descontroladas, especies invasoras, contaminación, entre otros, han incrementado la presión sobre la biodiversidad. A todo esto se añade en años recientes los impactos del cambio climático global.

Evidencia acumulada en los últimos años han documentado múltiples impactos bióticos en respuesta al cambio climático antrópico (Parmesan, 1996; Bellard et al., 2012; García et al., 2014). El incremento en las temperaturas globales y los cambios en el clima han originado alteraciones ecológicas en la biodiversidad (Walther et al., 2002; Parmesan, 2006; Cheng et al., 2011; Larsen et al., 2011). Estos impactos actúan a todos los niveles de organización biológica - de poblaciones a biomas, y pueden ser de índole espacial o temporal (Timaná, 2014), incluyendo cambios en la distribución de las especies, llegando de ese modo a causar profundos efectos ecosistémicos y, por ende, en los servicios que estos ofrecen a los humanos.

Frente a este reto, surge la necesidad de desarrollar técnicas analíticas que nos permitan evaluar la distribución real, potencial y futura de la biodiversidad.

Una línea de investigación de análisis de los patrones espaciales de presencia y ausencia de especies, y que se ha expandido exponencialmente en los últimos 15 ańos, son los modelos de distribución de especies MDE (Guisan y Zimmermann, 2000; Araújo y Guisan 2006; Austin 2002; Elith y Leathwick, 2009; Mateo et al., 2011; Peterson y Lieberman, 2012). Los MDE son representaciones cartográficas que muestran la idoneidad del espacio geográfico para la presencia de una especie en función 
a las variables ambientales utilizadas para generar dicha representación. Se basa en la asociación entre las observaciones georreferenciadas de la ocurrencia o abundancia de especies con varios predictores ambientales (clima, suelo, topografía, entre otros), usando una gama de métodos estadísticos (Pearson 2007; Pliscoff y Fuentes-Castillo 2011; Franklin et al., 2013). El objetivo de este artículo es entonces, esbozar las técnicas de distribución de especies y ofrecer un par de ejemplos ilustrativos de cómo esta herramienta puede ser utilizada para evaluar el impacto potencial del cambio climático en la biodiversidad peruana.

Se han desarrollado varias técnicas de modelamiento, pero, en esencia, todas estas establecen una relación matemática o estadística entre la distribución conocida de un organismo y los predictores ambientales utilizados (Franklin 2009, Franklin et al., 2013). Los predictores pueden ser climáticos, geológicos, topográficos, entre otros, y se espera que con algunos de estos, ya sea individualmente o en combinación, se puedan definir los factores que definan las condiciones ambientales favorables para la presencia de una especie (Guisan y Zimmermann, 2000; Mateo et al., 2011).

El progreso de los MDE está estrechamente relacionado al desarrollo y posterior sinergia de dos aspectos de la investigación biogeográfica: los Sistemas de Información Geográfica (SIG) y lo que se conoce como informática de la biodiversidad (Graham et al., 2004; Canhos et al., 2004; Anderson, 2012). Efectivamente, en las últimas dos décadas muchos herbarios y museos de historia natural alrededor del mundo han puesto a disposición de la comunidad científica internacional la información, en la forma de localidades, de sus colecciones biológicas, facilitando de ese modo el acceso a uno de los dos componentes básicos para el modelamiento de la distribución de especies (Anderson 2012). Una de las principales plataformas electrónicas disponibles en la actualidad es el Global Biodiversity Information Facility, o GBIF (www.gbif.org; Flemons et al., 2007; Telenius, 2011), el cual pone a disposición de sus usuarios más de 500 millones de datos de ocurrencia correspondientes a más de un millón y medio de especies en todos los niveles taxonómicos.

Aprovechando este cúmulo de información disponible sobre la distribución de especies, aunado con el desarrollo y acceso a modelos climáticos globales y regionales (ej. WorldClim; Hijmans et al., 2005) y la generalización de los sistemas de información geográfica (SIG), el modelamiento espacial se ha convertido en una de las herramientas más poderosas para los biogeógrafos e investigadores en conservación, lo que ha permitido examinar con mayor precisión el rango de las especies sobre la Tierra. Sus aplicaciones son muy numerosas y abarcan desde análisis de endemismo regional (Young, 2007; Young et al., 2009; Swenson et al., 2012), análisis de distribución de especies en el pasado (Varela et al., 2011), análisis de riesgo por especies invasoras (Robinson et al., 2010), descubrimiento de especies raras (Särkinen et al., 2013) entre muchos otros. 


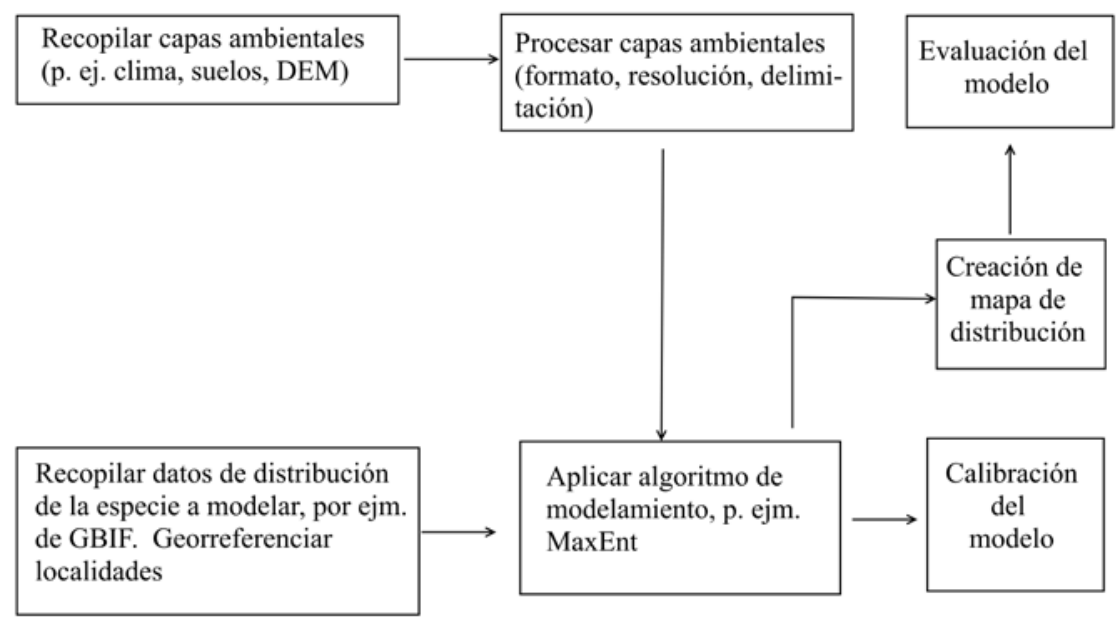

Figura 1. Diagrama de flujo simplificado de los pasos requeridos para el modelamiento de especies

Una de las aplicaciones directamente relacionada con esta propuesta es el análisis de cambios de rango de distribución en respuesta al cambio climático (Beaumontet al., 2008; Thuiller, 2007; Tovar et al., 2013; Costion et al., 2015; Timaná y Cuentas, 2015). Basados en uso comparativo de modelos climáticos del pasado, presente y futuro (disponibles por ejemplo a través de WorldClim, www.worldclim.org y del CCAFS-CGIAR, www.ccafs-climate.org) y bajo ciertas restricciones conceptuales (Anderson, 2013), estos modelos permiten estimar la distribución potencial para el tiempo en que el clima ha sido modelado. Siguiendo esta estrategia se ha modelado la distribución futura de especies en varios ámbitos geográficos, tales como lémures en Madagascar (Brown y Yoder, 2015), mamíferos en México (Aguado y Escalante, 2015), anfibios en Nueva Zelanda (Fouquet et al., 2010), hasta la distribución de biomas andinos (Tovar et al., 2013), entre muchos otros (ver Mateo et al., 2011 para una lista extensa de aplicaciones).

\section{Técnicas de modelamiento}

Tal como lo detallan Pliscoff y Fuentes-Castillo (2011), se pueden reconocer cuatro clases de técnicas de modelamiento de distribución de especies: modelos estadísticos de regresión, métodos de clasificación, métodos de «sobre» climático y, finalmente, aquellos métodos basados en algoritmos específicos como GARP y MaxEnt (ver Franklin 2009 para una discusión detallada de estos métodos). En este artículo presentaremos la aplicación del modelamiento de especies con MaxEnt por ser el más ampliamente usado en la literatura. 


\section{MaxEnt y el Principio de Máxima Entropía}

Primeramente debe aclararse que el término MaxEnt se emplea en dos contextos diferentes pero relacionados: a) para referirse al método estadístico basado en el principio de máxima entropía y b) para denotar el programa de cómputo MaxEnt diseńado por Phillips et al. (Phillips et al., 2004; Phillips et al., 2006), el cual aplica el principio previamente citado en el modelamiento de distribución de especies.

El principio de máxima entropía (PME) tiene sus raíces en el trabajo de Claude Shannon sobre la teoría matemática de la comunicación (Shannon 1948) y luego el de Jaynes en el campo de mecánica estadística (Jaynes, 1957. Ver Pierce 1980, para una detallada reseña y discusión sobre el PME en el contexto de teoría de la información). Tal como lo indican Harte y Newman (2014), MaxEnt es un algoritmo de inferencia estadística ampliamente utilizado en tópicos tan diversos como termodinámica, economía, ciencias forenses, análisis de imágenes, procesamiento de señales y recientemente en ecología y biogeografía. La idea básica de MaxEnt es la de estimar una distribución de probabilidad dada usando aquella distribución más uniforme (es decir, una en que la entropía sea máxima) teniendo en cuenta las restricciones dadas por la información disponible del fenómeno estudiado. En el contexto que aquí nos interesa, el de la biogeografía predictiva, usamos Maxent para estimar la distribución de puntos de ocurrencia más uniforme posible en el área de estudio tomando en consideración las restricciones impuestas por las variables ambientales (clima, suelos, entre otros). Tal como lo indica Pearson (2007): «la distribución (geográfica) estimada debe concordar con todo lo que se sabe (o inferida a partir de las condiciones ambientales donde la especie ha sido observada) pero debería evitar hacer supuestos que no son apoyados por los datos».

El software MaxEnt es un programa de libre disponibilidad (descargable de https:// www.cs.princeton.edu/ $\sim$ schapire/maxent) presentado por Steven Phillips, Robert Anderson, Robert Schapire y Miroslav Dudík (Phillips et al., 2004; 2006) el cual implementa el principio de máxima entropía que busca encontrar la distribución con la mayor uniformidad posible, dadas las limitaciones impuestas por la información disponible sobre la distribución observada (puntos de colecta) de las especies y las condiciones ambientales en el área de estudio (Martínez, 2010). Aunque existen diferentes técnicas y algoritmos de modelamiento basadas en múltiples técnicas estadísticas (Elith et al., 2006; Elith y Leathwick, 2009; Mateo et al., 2011), varios estudios han demostrado la superioridad de MaxEnt en el modelamiento espacial de especies, más aún cuando hay un limitado número de puntos de colecta (Phillips et al., 2006; Tognelli y Roig-Junent, 2009). Este programa ha sido utilizado en una amplia gama de aplicaciones tales como predicciones en la distribución de especies invasoras, predicción de diversidad y riqueza de especies, determinar paleodistribuciones, entre muchos otros (Elith et al., 2011). 


\section{EJEMPLOS ILUSTRATIVOS DE MODELAMIENTO}

En esta sección presentamos dos ejemplos ilustrativos de modelamiento de especies utilizando el algoritmo de MaxEnt. Se modelaron dos especies vegetales, una de la costa norte peruana, y la segunda de las zonas altoandinas de Chile y Bolivia. En ambos casos se ha modelado la distribución potencial presente así como un pronóstico de la distribución potencial futura bajo cierto escenario de cambio climático (RPG).

\section{Materiales y métodos}

\section{a) Las especies}

El algarrobo, conocido botánicamente como Prosopis pallida H.B.K., es un árbol leguminoso que crece y se desarrolla en zonas áridas y semiáridas. Su distribución se restringe a países de Sudamérica como Perú, Ecuador y Colombia, particularmente en zonas costeras peruanas en altitudes bajas y medias (Skolmen, 1990; Prokopiuk et al., 2000). Es una especie arbórea de hasta $20 \mathrm{~m}$ de altura —en condiciones favorables- y cuenta con raíces de hasta $60 \mathrm{~m}$ de profundidad para acceder a las aguas subterráneas, lo que les permite ocupar sectores donde escasea el agua, siendo dominantes en depresiones secas o estacionalmente secas. Su período de floración se da normalmente entre diciembre a febrero, mientras que su período de fructificación es de junio a agosto. Es muy adaptable en zonas con pocas precipitaciones, siendo menores a $200 \mathrm{~mm}$ por año generalmente. Sin embargo, en las épocas de lluvias suele regenerarse y se recubren áreas que han sido anteriormente deforestadas. Por otro lado, la temperatura ideal para su crecimiento está entre 20 a $30^{\circ} \mathrm{C}$, siendo susceptibles a los eventos de heladas o temperaturas muy bajas. Finalmente, es una especie muy valorada como leña y carbón. Se considera además como árbol de sombra cerca de las viviendas rurales (Prokopiuk, et al., 2000; Marcelo et al., 2010; Dostert et al., 2012).

Pycnophyllum spathulatum Mattf. es un arbusto sufrútice, miembro de la familia Caryophyllaceae. El género Pycnophyllum consiste de nueve especies, las cuales se distribuyen desde el centro del Perú hasta el norte de la Argentina (Timaná 2005; Beck et al., 2014). Las especies en este género se distribuyen en las partes más altas de los Andes, usualmente por encima de los $4000 \mathrm{~m}$ en Perú y bordeando los $5000 \mathrm{~m}$ en Bolivia, formando así un elemento endémico a esta franja altitudinal. P. spathulatum ha sido reportado en la puna andina del norte de Chile (Antofagasta) y el suroeste de Bolivia (Oruro, Potosí), bajo extremos de temperatura; crece formando densas almohadillas de 30-80 cm de diámetro. Al ser una especie dioca con frutos en utrículo (Timaná 2005), muestra una muy limitada capacidad de dispersión. 


\section{b) Modelamiento}

Tal como se mencionó anteriormente, todo proceso de modelamiento de especies requiere de dos clases de datos: datos de presencia del organismo a modelar y las variables predictores ambientales (Guisan y Zimmermann, 2000; Elith y Leathwick, 2009)

a) Datos de presencia de la especie: Son las localidades georreferenciadas, siendo su fuente de información principalmente herbarios o museos que sirven como depositarios de colecciones de especímenes y con su respectiva información geoespacial (Soberon y Peterson, 2004). Los datos de las localidades del Prosopis pallida fueron obtenidos a partir de una investigación elaborada previamente (Cuentas, 2015), al igual que las localidades de Pycnophyllum spathulatum (Timaná, 2005). Las principales bases de datos digitales utilizadas y de libre acceso para obtener este tipo de información fueron el GBIF y TROPICOS (www.tropicos.org) bajo la administración del Jardín Botánico de Missouri, St. Louis. Otras fuentes fueron los herbarios locales (Herbario de la Universidad Nacional Agraria La Molina) y diversas publicaciones científicas. Las localidades de P. spathulatum fueron recolectadas de aproximadamente 60 herbarios internacionales. Luego de recolectar las localidades suficientes para el modelamiento se procedió a un minucioso control de calidad para verificar y/o corregir las coordenadas geográficas de los puntos de colecta.

b) Variables o predictores ambientales: Siguiendo la metodología de Hernandez (2007), se seleccionaron datos ambientales para SIG que son ecológicamente relevantes para las zonas de estudio y las especies focales seleccionadas. Para esta investigación se utilizaron las variables bioclimáticas que se encuentran en la página web de WorldClim. En un primer ensayo de modelamiento se trabajaron las 19 variables bioclimáticas — cuyos datos comprenden el período de 1950 al 2000- y la variable de altitud, las cuales tienen una resolución máxima de $1 \mathrm{~km}$ (Tabla 1). WorldClim ofrece además proyecciones climáticas a partir de modelos climáticos globales (GCM) de cuatro vías de concentración representativas (RCP). Estas son las más recientes proyecciones climáticas globales que se utilizan en el Quinto Informe del IPCC (IPCC, 2014). Para este estudio se seleccionaron interpolaciones en alta resolución (aprox. $1 \mathrm{~km}$ ) del modelo global CCSM4 (Community Earth System Model 4; Hurrell et al., 2013) bajo el RCP 8.5, un escenario pesimista de alta emisión de gases de invernadero (Moss et al., 2010; Riahi et al., 2011) para el año 2050. 
Tabla 1. Variables bioclimáticas de WorldClim

\begin{tabular}{ll}
\hline Código & Variable bioclimática \\
\hline BIO1 & Temperatura promedio anual \\
BIO2 & Rango medio diurno (temp máx - temp min; promedio mensual \\
BIO3 & Isotermalidad (BIO1/BIO7)* 100 \\
BIO4 & Estacionalidad en temperatura (coeficiente de variación) \\
BIO5 & Temperatura máxima del período más caliente \\
BIO6 & Temperatura mínima del período más frío \\
BIO7 & Rango anual de temperatura (BIO5-BIO6) \\
BIO8 & Temperatura media en el trimestre más lluvioso \\
BIO9 & Temperatura promedio en el trimestre más seco \\
BIO10 & Temperatura promedio en el trimestre más caluroso \\
BIO11 & Temperatura promedio en el trimestre más frío \\
BIO12 & Precipitación anual \\
BIO13 & Precipitación en el período más lluvioso \\
BIO14 & Precipitación en el período más seco \\
BIO15 & Estacionalidad de la precipitación (Coeficiente de variación) \\
BIO16 & Precipitación en el trimestre más lluvioso \\
BIO17 & Precipitación en el trimestre más seco \\
BIO18 & Precipitación en el trimestre más caluroso \\
BIO19 & Precipitación en el trimestre más frío \\
\hline
\end{tabular}

El proceso para el modelamiento con MaxEnt parte de la preparación de los datos de presencia de la especie y de las variables ambientales. Para el primero se creó un archivo Excel con únicamente tres columnas en el siguiente orden: especie, longitud y latitud. Luego se verificaron que las localidades estén dentro del área de estudio, lo cual se realizó con ArcMap. Posteriormente se cambió el formato Excel (xlsx) a uno delimitado por comas (csv). Para la preparación de las variables ambientales, estas fueron recortadas al área de estudio y finalmente se convierten a formato ASCII (asc), lo cual es un requisito necesario para que estos datos sean procesados por el programa MaxEnt.

El segundo paso es el modelamiento en sí en el programa MaxEnt, versión 3.3.3.k. La interface de MaxEnt ofrece tres áreas de trabajo (Figura 2): el panel izquierdo (Samples), donde se adicional el archivo en formato csv que contiene las coordenadas de la especie a modelar, un panel derecho (Environmental layers) donde se incorporan las variables predictoras y una región inferior donde se procesa la configuración del análisis. 
Para ello, en la sección Samples se agregó el archivo csv de la especie correspondiente a modelar. En la sección de Environmental layers se agregaron todos los archivos ASCII derivados de los rásters de las variables ambientales, y en Output directory se ubicó la carpeta donde se guardan todos los resultados. Debe resaltarse que, para este estudio estamos modelando dos especies bajo dos tiempos diferentes (presente y futuro). A continuación se definieron algunos parámetros de MaxEnt tales como activar las opciones Make pictures of predictions y Do jackknife to measure variable importance. Además, en Output format se colocó la opción Logistic. Por otro lado, en la pestaña Settings se verificó que esté activada la opción Remove duplicate presence records y en Replicate run type se eligió la opción Crossvalidate. Luego de tener todas las opciones activadas y los archivos en el lugar correspondiente, se ejecuta el análisis con la opción Run. Finalmente, para la proyección por cambio climático se agregó la carpeta con las variables bioclimáticas futuras en formato ASCII en la casilla de Projection layers directory/file, con los mismos nombres que las variables presentes.

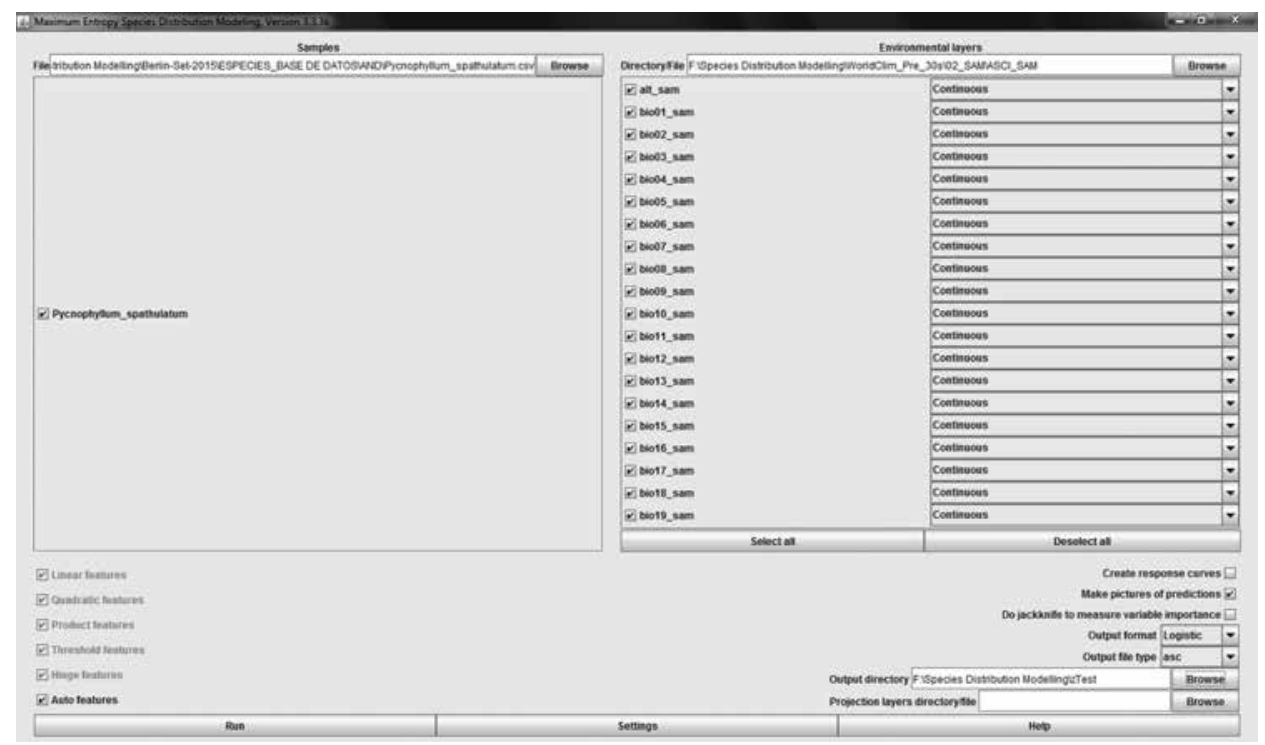

Figura 2. Interface de trabajo en MaxEnt

Para ambas especies se realizó una primera ejecución de MaxEnt con las 19 variables bioclimáticas y la altitud. A partir de los resultados de la prueba de Jackknife, que determina qué variables son más importantes para el modelo, se descartaron algunas de estas dejando las de mayor ganancia para realizar una segunda ejecución (Tabla 2). 
Tabla 2. Variables seleccionadas para el modelamiento de cada una de las especies

\begin{tabular}{|c|c|c|}
\hline Variable & Prosopis pallida & Pycnophyllum spathulatum \\
\hline BIO1 & X & $X$ \\
\hline \multicolumn{3}{|l|}{$\mathrm{BIO} 2$} \\
\hline \multicolumn{3}{|l|}{$\mathrm{BIO} 3$} \\
\hline $\mathrm{BIO} 4$ & $\mathrm{X}$ & \\
\hline $\mathrm{BIO} 5$ & $\mathrm{X}$ & $\mathrm{X}$ \\
\hline $\mathrm{BIO6}$ & $\mathrm{X}$ & $\mathrm{X}$ \\
\hline BIO7 & $\mathrm{X}$ & \\
\hline BIO8 & $\mathrm{X}$ & $\mathrm{X}$ \\
\hline BIO9 & $\mathrm{X}$ & $\mathrm{X}$ \\
\hline $\mathrm{BIO} 10$ & $\mathrm{X}$ & $\mathrm{X}$ \\
\hline BIO11 & & $\mathrm{X}$ \\
\hline $\mathrm{BIO} 12$ & $\mathrm{X}$ & $\mathrm{X}$ \\
\hline $\mathrm{BIO} 13$ & $\mathrm{X}$ & \\
\hline \multicolumn{3}{|l|}{ BIO14 } \\
\hline $\mathrm{BIO} 15$ & & $\mathrm{X}$ \\
\hline BIO16 & $\mathrm{X}$ & \\
\hline \multicolumn{3}{|l|}{ BIO17 } \\
\hline \multicolumn{3}{|l|}{ BIO18 } \\
\hline \multicolumn{3}{|l|}{ BIO19 } \\
\hline Altitud & $\mathrm{X}$ & $\mathrm{X}$ \\
\hline
\end{tabular}

\section{Resultados}

MaxEnt ofrece varios tipos de resultados, tales como las pruebas necesarias para determinar la validez estadística de los modelos encontrados. Además muestra la contribución de cada variable al modelamiento (Martínez, 2010; Phillips, 2010). MaxEnt produce además un mapa, en formato png, el cual muestra, «en bruto», los resultados del modelamiento. Dicho mapa usa colores para indicar la probabilidad que las condiciones son adecuadas: el rojo indica una alta probabilidad de condiciones adecuadas para la especie, verde indica las condiciones típicas de aquellos lugares donde la especie se encuentra, y sombras más tenues de azul indican una baja probabilidad de condiciones adecuadas (Phillips, 2010). Además de este, MaxEnt produce un archivo en formato asc, el cual puede ser procesado en un software de SIG, tal como se procedió en este 
estudio. En la figuras 3 y 4 las áreas de color naranja a rojas representan espacios con alta probabilidad de idoneidad ecológica para la especie $(>0,7)$, mientras que las zonas de color amarilla tienen una media a menor probabilidad. Posteriormente se cuantificó el área de mayor idoneidad potencial $(0,7-1,0)$ para cada una de las especies tanto para el modelamiento con datos climáticos presentes como futuros (Tabla 3).

Los resultados del modelamiento de Pycnophyllum spathulatum muestra primeramente que, basados en los datos climáticos actuales (Figura 3a) hay una mayor idoneidad ecológica en lo que correspondería a la puna xerofítica del norte de Chile (Regiones I y XV), el oeste de Bolivia (Oruro, Potosí) y el noreste de Argentina (Jujuy, Salta). Debe notarse que en esta última localidad no se ha reportado la presencia de esta especie. La extensión total de áreas idóneas, basado en variables climáticas actuales, es de 6708804.71 ha (Tabla 3). Al examinar el mismo parámetro proyectado para el año 2050 bajo el RCP 8.5 se puede observar que dicha extensión disminuye drásticamente a 945642.69 ha, es decir, una reducción de casi $85 \%$. El ámbito geográfico es aproximadamente el mismo (la trifrontera mencionada anteriormente), aunque se puede apreciar una marcada reducción en el norte argentino como en el lado boliviano.

Tabla 3. Extensión de las áreas potenciales de distribución, presente y futura de las especies modeladas

\begin{tabular}{lcc}
\hline Especie & $\begin{array}{c}\text { Área potencial actual } \\
(\mathbf{0 . 7}-\mathbf{1})\end{array}$ & $\begin{array}{c}\text { Área potencial futura } \\
(\mathbf{0 . 7}-\mathbf{1})(\text { RCP 8.5) }\end{array}$ \\
\hline Prosopis pallida & $\begin{array}{c}60513,87 \mathrm{ha} \\
\text { Pycnophyllum spathulatum }\end{array}$ & $653717,18 \mathrm{ha}$ \\
\hline
\end{tabular}

En el caso de Prosopis pallida, este se distribuye desde Piura hasta la parte norte de La Libertad, concentrando su presencia en Lambayeque, donde se ubican los bosques de algarrobo más densos en el Perú (Figura 4a). Se muestran, además, que las mayores probabilidades de hábitats idóneos en el departamento de Lambayeque están en las áreas designadas como unidades de conservación: Santuario Histórico Bosque de Pómac, Refugio de Vida Silvestre Laquipampa, ACR Huacrupe La Calera, ACR Moyán Palacios y ACP Chaparrí. La extensión total es de 60513,87 ha (distribución potencial presente. Tabla 3). Sin embargo, la potencialidad del hábitat se ve bajo amenaza por la deforestación, invasiones y expansión agrícola. Si bien se encuentra la mayor probabilidad en las zonas de protección, hay otros espacios cuyo potencial es alto y sus condiciones son favorables para el desarrollo de la especie, siendo la variable de mayor importancia la altitud. 
A partir de los escenarios climáticos futuros se realizó el modelo de distribución de esta especie para el año 2050 en donde se puede observar que $P$. pallida incrementaría su rango de distribución (653717.18 ha), extendiéndose a un mayor territorio en los departamentos de Tumbes, Piura y La Libertad. Esto significaría que el cambio climático vendría a ser positivo para la especie debido a que algunas de las variables de mayor importancia para el modelo futuro, además de la altitud, son los derivados de la variables de precipitación — precipitación en el trimestre más caluroso (BIO 18), precipitación en el trimestres más lluvioso (BIO 16) y precipitación anual (BIO 12)— lo que permitiría la regeneración de los bosques secos de algarrobo.

\section{Discusión}

Los ecosistemas de alta montaña (incluyendo aquí parajes altoandinos en Sudamérica y los alpinos en un sentido global), por encima de la línea arbórea han sido reconocidos como los más frágiles ante los efectos del cambio climático (Walther et al., 2002; Grabherr et al., 2010). Esta fragilidad es el resultado por un lado, del retroceso glaciar y por otro, de las características intrínsecas de los hábitats alpinos. La reducción observada en la masa glaciar conduce a la creación de nuevas áreas potencialmente colonizables por especies alpinas (ej. Seimon et al., 2007) y, por ende, a un desplazamiento espacial de los ecosistemas adyacentes, tal como se ha documentado en los boques de Polylepis (Rosaceae) en los Andes de Ancash, Perú (Cáceres, 2007). Aunque todos los ecosistemas terrestres son afectados por el cambio climático, los de la biozona de alta montańa son considerados particularmente sensibles a dicho calentamiento puesto que están condicionados por las bajas temperaturas (Grabherr et al.,1994; Pauli et al., 2012).

Uno de los patrones de desplazamiento observados en respuesta al cambio climático es la migración de especies, ya sea hacia latitudes ahora más cálidas, o en el caso de montañas, de zonas de laderas bajas, hacia las zonas más altas, lo que se ha denominado «migración cuesta arriba» (Parmesan 2006; Laurance et al., 2011). Las especies en las partes más altas de cadenas montañosas son más susceptibles a extinguirse debido a que, a diferencia de especies de zonas bajas, aquellas no cuentan con espacios adicionales hacia donde migrar. El área disponible para colonización disminuye con la elevación lo cual acrecienta el problema (Larsen et al., 2011; Laurance et al., 2011). Así pues, los resultados obtenidos en el caso de Pycnophyllum spathulatum son concordantes con la tendencia global que muestra una reducción y, en ciertos casos, extinción local de especies en otras regiones montañosas (Pauli et al., 2012; Tovar et al., 2013; Costion et al., 2015; Timaná y Cuentas, 2015). 


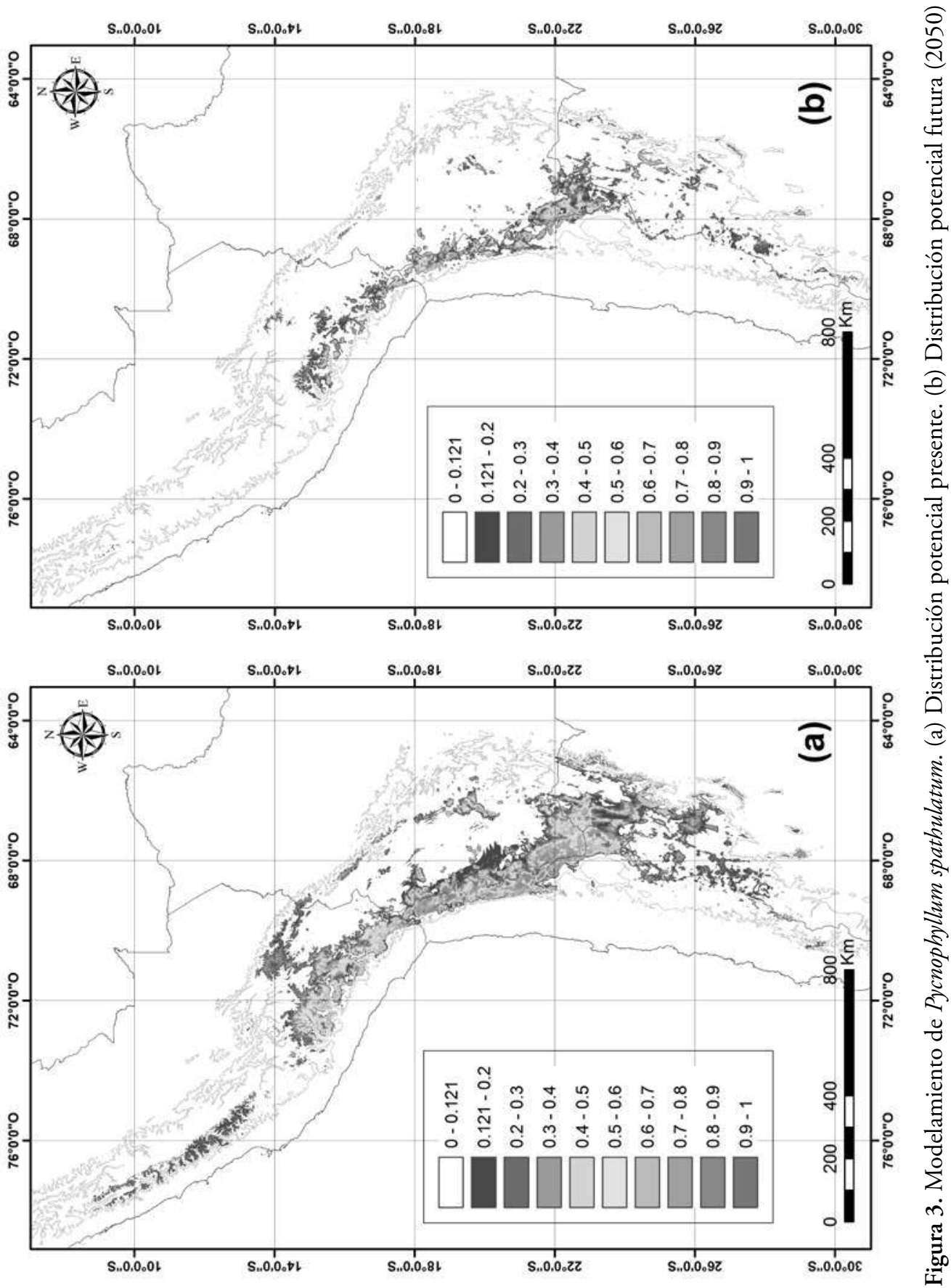




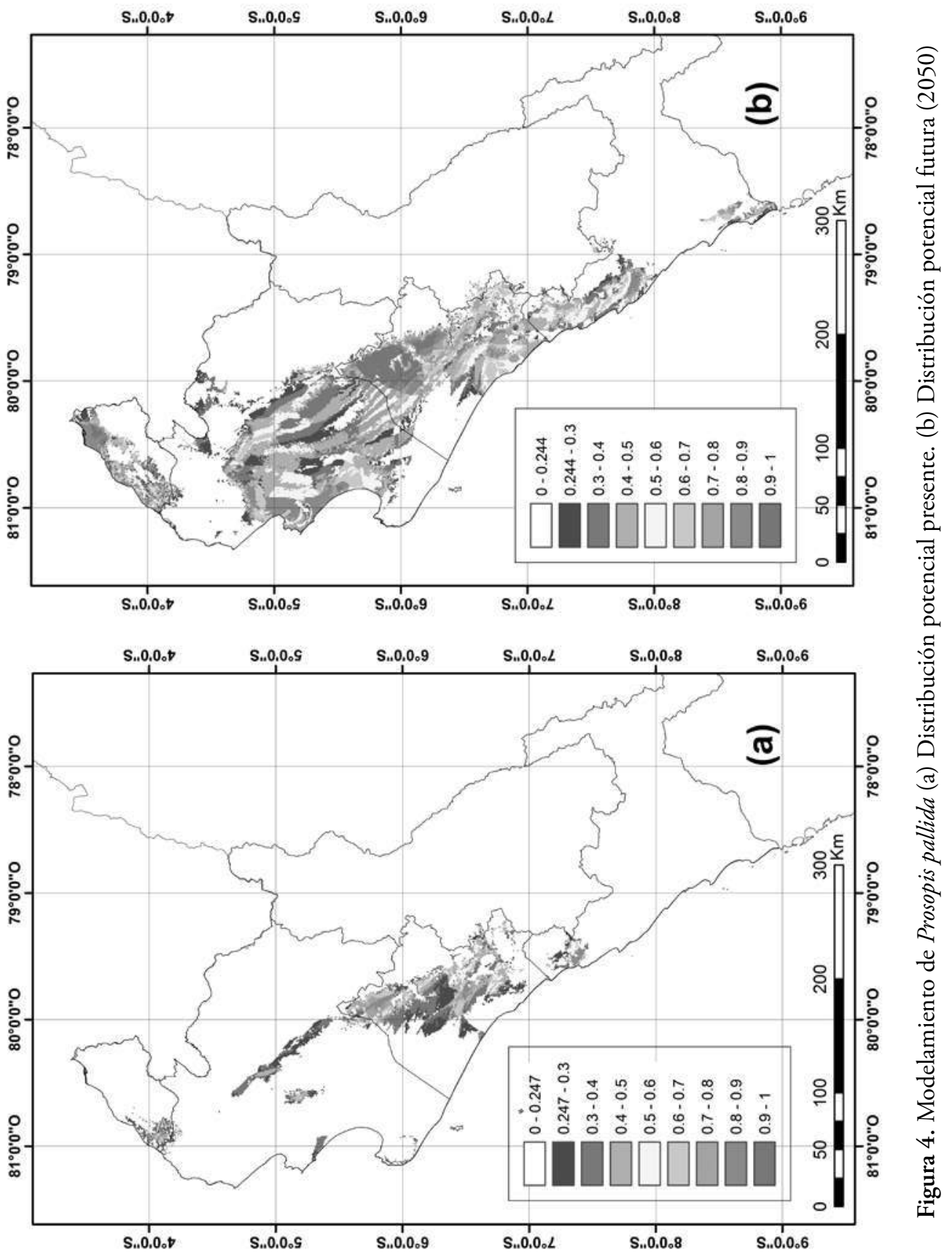


En el caso del algarrobo, los resultados muestran una tendencia opuesta. Según la importancia que representó cada variable, se supone que el incremento de la temperatura y de las precipitaciones por causa de los GEI sería favorable para el crecimiento de los bosques de algarrobo, pues el modelo muestra una expansión a futuro. Sin embargo, es importante considerar que, a diferencia del nuestro, existen modelos que toman en cuenta procesos de deforestación y cambio de uso de suelo, por lo que podrían haber resultados predictivos diferentes, sobre todo porque estos están bajo una situación actual en donde la tala es muy constante e intensa. Modelos como por ejemplo DIMA (Dynamic Integrated Model of Forestry and Alternative Land Use) podrían ser de gran utilidad para este caso pues toma en cuenta procesos forestales como deforestación, reforestación y manejo forestal (Riahi et al., 2011), sin embargo no se cuentan con los escenarios climáticos para ser aplicados. Es por tanto que para una zona como la costa norte peruana se requieren de modelos regionales orientados a las características de dicho ámbito geográfico, en este caso los bosques secos, sin embargo solo se cuentan con los modelos climáticos globales (GCM), siendo una limitación en el modelamiento de especies.

Como conclusión, la distribución potencial de algarrobo (Prosopis pallida) se extiende en toda la costa norte, demostrando que hay tierras potenciales para albergarlos y que en un futuro puedan convertirse en tierras de protección. El departamento de Lambayeque tiene la mayor presencia de algarrobos, mientras que en el escenario futuro tanto Piura, La Libertad y Tumbes contarán con una distribución similar por impacto del cambio climático.

Si bien el cambio climático beneficiaría a la especie a futuro, se debe considerar que el modelo se basa en el aumento de GEI por demanda de energía debido al crecimiento poblacional y decrecimiento del PBI, mas no se toma en cuenta totalmente procesos como deforestación o cambios en los usos de suelo por lo que los resultados de la distribución, si se utilizaran otros modelos basados en dichos procesos, podrían ser diferentes.

Para modelar de manera exitosa la especie del algarrobo se requieren adicionalmente variables de cobertura vegetal, de suelo y napa freática de la zona, siendo los factores más importantes para su distribución la altitud, la vegetación natural y la presencia de aguas subterráneas, por lo que es necesario generar un mapa y base de datos que puedan utilizarse a nivel regional y nacional, y que puedan obtenerse tanto del Minam, Minagri o Ingemmet. 


\section{REFERENCIAS BIBLIOGRÁFICAS}

Aguado, O. \& Escalante, T. (2015). Cambios en los patrones de endemismo de los mamíferos terrestres de México por el calentamiento global. Revista Mexicana de Biodiversidad, 86, 99-110. http://dx.doi.org/10.7550/rmb.46637

Anderson, R. P. (2012). Harnessing the world's biodiversity data: promise and peril in ecological niche modeling of species distributions. Annals of the New York Academy of Sciences, 1260, 66-80. http://dx.doi.org/10.1111/j.1749-6632.2011.06440.x

Anderson, R. P. (2013). A framework for using niche models to estimate impacts of climate change on species distributions. Annals of the New York Academy of Sciences, 1297(1), 8-28. http://dx.doi.org/10.1111/nyas.12264

Araújo, M. B. \& Guisan, A. (2006). Five (or so) challenges for species distribution modelling. Journal of Biogeography, 33(10), 1677-1688. http://dx.doi.org/10.1111/ j.1365-2699.2006.01584.x

Austin, M. (2002). Spatial prediction of species distribution: an interface between ecological theory and statistical modelling. Ecological Modelling, 157(2-3), 101-118. http:// dx.doi.org/10.1016/S0304-3800(02)00205-3

Beaumont, L. J., Hughes, L. \& Pitman, A. J. (2008). Why is the choice of future climate scenarios for species distribution modelling important? Ecology Letters, 11, 1135-1146. http://dx.doi.org/10.1111/j.1461-0248.2008.01231.x

Beck, S., Rojas, P., Romero, C. y Timaná, M. E. (2014). Caryophyllaceae. En Jorgensen, P., Nee, M. y Beck, S., Catálogo de Plantas Vasculares de Bolivia (pp. 490-497). St. Louis, Missouri: Missouri Botanical Garden Press.

Bellard, C., Bertelsmeier, C., Leadley, P., Thuiller, W. y Courchamp, F. (2012), Impacts of climate change on the future of biodiversity. Ecology Letters 15, 365-377. http:// dx.doi.org/10.1111/j.1461-0248.2011.01736.x

Brown, J. L. \& Yoder, A. D. (2015). Shifting ranges and conservation challenges for lemurs in the face of climate change. Ecology and Evolution, 5(6), 1131-1142. http://dx.doi. org/10.1002/ece3.1418

Cáceres, R. (2007). Movimiento límite de los bosques de Polylepis en relación al retroceso glaciar en la microcuenca Quillcay-Ancash. Acta Universitatis Carolinae, 1-2, 183-206.

Canhos, V., Sousa, S., Giovanni, R. \& Canhos, D. (2004). Global biodiversity informatics: setting the scene for a New World of ecological modeling. Biodiversity Informatics, $1(1), 1-13$.

Cheng, I.C., Hill, J.K., Ohlemuller, R., Roy, D.B., Thomas, C.D. (2011). Rapid range shift of species associated with high levels of climate warming. Science, 333, 10241026. http://dx.doi.org/10.1126/science. 1206432

Costion, C. M., Simpson, L., Pert, P. L., Carlsen, M. M., John Kress, W. \& Crayn, D. (2015). Will tropical mountaintop plant species survive climate change? Identifying 
Timaná y Cuentas / Biogeografía predictiva: técnicas de modelamiento de distribución de especies...

key knowledge gaps using species distribution modelling in Australia. Biological Conservation, 191, 322-330. http://dx.doi.org/10.1016/j.biocon.2015.07.022

Cuentas, M. A. (2015). Revalorizando el bosque seco de algarrobo: estudio y análisis de la biodiversidad, distribución y conservación de los bosques secos en Lambayeque. Tesis de Licenciatura. Lima: Pontificia Universidad Católica del Perú.

Dostert, N., Roque, J., Cano, A., La Torre, M. \& Weigend, M. (2012). Hoja botánica: Algarrobo. Prosopis pallida (Humb. \& Bonpl. ex Willd.) Kunth. Lima: Museo de Historial Natural, Universidad Mayor de San Marcos.

Ehrlen, J. \& Morris, W. F. (2015). Predicting changes in the distribution and abundance of species under environmental change. Ecology Letters, 18(3), 303-314. http://dx.doi. org/10.1111/ele.12410

Elith, J., Graham, C. H., Anderson, R. P., Dudi, M., Hijmans, R. J., Huettmann, F., Zimmermann, N. E. (2006). Novel methods improve prediction of species distributions from occurrence data. Ecography, 29(2), 129-151. http://dx.doi.org/10.1111/ j.2006.0906-7590.04596.x

Elith, J. \& Leathwick, J. R. (2009). Species Distribution Models: Ecological Explanation and Prediction across Space and Time. Annual Review of Ecology, Evolution, and Systematics, 40(1), 677-697. http://dx.doi.org/10.1146/annurev.ecolsys.110308.120159

Elith, J., Phillips, S. J., Hastie, T., Dudík, M., Chee, Y. E. \& Yates, C. J. (2011). A statistical explanation of MaxEnt for ecologists. Diversity and Distributions, 17(1), 43-57. http://dx.doi.org/10.1111/j.1472-4642.2010.00725.x

Flemons, P., Guralnick, R., Krieger, J., Ranipeta, A. \& Neufeld, D. (2007). A web-based GIS tool for exploring the world's biodiversity: The Global Biodiversity Information Facility Mapping and Analysis Portal Application (GBIF-MAPA). Ecological Informatics, 2(1), 49-60. http://dx.doi.org/10.1016/j.ecoinf.2007.03.004

Fouquet, A., Ficetola, G. F., Haigh, A. \& Gemmell, N. (2010). Using ecological niche modelling to infer past, present and future environmental suitability for Leiopelma hochstetteri, an endangered New Zealand native frog. Biological Conservation, 143(6), 1375-1384. http://dx.doi.org/10.1016/j.biocon.2010.03.012

Franklin, J. (2009). Mapping Species Distribution. Spatial Inference and Prediction. Cambridge: Cambridge University Press.

Franklin, J., Davis, F. W., Ikegami, M., Syphard, A. D., Flint, L. E., Flint, A. L. \& Hannah, L. (2013). Modeling plant species distributions under future climates: how fine scale do climate projections need to be? Global Change Biology, 19(2), 473-483. http:// dx.doi.org/10.1111/gcb.12051

García, R. A., Cabeza, M., Rahbek, C. \& Araújo, M. B. (2014). Multiple dimensions of climate change and their implications for biodiversity. Science, 344(6183), 1247579. http://dx.doi.org/10.1126/science.1247579 
Grabherr, G., Gottfried, M., Pauli, H. (1994). Climate effects on mountain plants. Nature, 369, 448. http://dx.doi.org/10.1038/369448a0

Grabherr, G., Gottfried, M. \& Pauli, H. (2010). Climate Change Impacts in Alpine Environments. Geography Compass, 4(8), 1133-1153.

Graham, C. H., Ferrier, S., Huettman, F., Moritz, C. \& Peterson, A. (2004). New developments in museum-based informatics and applications in biodiversity analysis. Trends in Ecology \& Evolution, 19(9), 497-503. http://dx.doi.org/10.1016/j.tree.2004.07.006

Guisan, A. \& Zimmermann, N. (2000). Predictive habitat distribution models in ecology. Ecological Modelling, 135, 147-186. http://dx.doi.org/10.1016/S0304$3800(00) 00354-9$

Harte, J. \& Newman, E. A. (2014). Maximum information entropy: A foundation for ecological theory. Trends in Ecology and Evolution, 29(7), 384-389. http://dx.doi. org/10.1016/j.tree.2014.04.009

Hernández, P. A. (2007). Métodos para crear los modelos de distribución. En Young, B.E. (Ed.), Distribución de las especies endémicas en la vertiente oriental de los Andes en Perú y Bolivia (pp. 13-17). Arlington: NatureServe.

Hijmans, R. J., Cameron, S. E., Parra, J. L., Jones, P. G. \& Jarvis, A. (2005). Very high resolution interpolated climate surfaces for global land areas. International Journal of Climatology, 25(15), 1965-1978. http://dx.doi.org/10.1002/joc.1276

Hurrell, J. W., Holland, M. M., Gent, P. R., Ghan, S., Kay, J. E., Kushner, P. J., Marshall, S. (2013). The Community Earth System Model: A Framework for Collaborative Research. Bulletin of the American Meteorological Society, 94(9), 1339-1360.

IPCC (2014) Climate Change 2014: Synthesis Report. Contribution of Working Groups I, II and III to the Fifth Assessment Report of the Intergovernmental Panel on Climate Change [Core Writing Team, R.K. Pachauri and L.A. Meyer (Eds.)]. IPCC, Geneva, Switzerland, 151.

Jaynes, E.T. (1957). Information theory and statistical mechanics. The Physical Review, 108(4), 620-630.

Larsen, T.H., Brehm, G., Navarrete, H., Franco, P., Gómez, H., Mena, J.L., Morales, V., Argollo, J., Blacutt, L. y Canhos, V. (2011). Range Shifts and Extinctions Driven by Climate Change in the Tropical Andes: Synthesis and Directions. En Herzog, S.K., Martinez, R., Jorgensen, P.M., Tiessen, H. (Eds.), Climate Change and Biodiversity in the Tropical Andes (pp. 47-67). São Paulo: Inter-American Institute for Global Change Research y Scientific Committee on Problems of the Environment (SCOPE).

Laurance, W.F., D.C. Useche, L.P. Shoo, S.K. Herzog, M. Kessler, F. Escobar, G. Brehm, J.C. Axmacher, I. C. Chen, L. Arellano G., P. Hietz, K. Fiedler, T. Pyrcz, J. Wolf, C.L. Merkord, C. Cardelús, A. Marshall, C. Ah-Peng, G.H. Aplet, M. del Coro A., W.J. Baker, J. Barone, C.A. Brühl, R.W. Bussmann, D. Cicuzza, G. Eilu, M.E. Favila, A. Hemp, C. Hemp, J. Homeier, R.B. Huey, J. Hurtado, J. Jankowski, G. Kattán, 
Timaná y Cuentas / Biogeografía predictiva: técnicas de modelamiento de distribución de especies...

J. Kluge, T. Krömer, D. Lees, M. Lehnert, J.T. Longino, J. Lovett, P.H. Martin, B. Patterson, R. Pearson, K.S.-H. Peh, B. Richardson, M. Richardson, M. Samways, F. Senbeta, T.B. Smith, T. Utteridge, J.E. Watkins, R. Wilson, S.E. Williams \& C.D. Thomas (2011). Global warming, elevational ranges and the vulnerability of tropical biota. Biological Conservation, 144, 548-557. http://dx.doi.org/10.1016/j. biocon.2010.10.010

Marcelo, J., Pennington, R., Reynel, C. \& Zevallos, P. (2010). Guía ilustrada de la flora leñosa de los bosques estacionalmente secos de Jaén, Perú. Lima: Royal Botanic Garden Edinburgh / Universidad Nacional Agraria La Molina.

Martínez, E. (2010). Apuntes sobre modelación de nichos ecológicos. México D.F.: Universidad Nacional Autónoma de México (UNAM).

Mateo, R., Felicísimo, Á. \& Muñoz, J. (2011). Modelos de distribución de especies: una revisión sintética. Revista Chilena de Historia Natural, 84, 217-240. http://dx.doi. org/10.4067/S0716-078X2011000200008

Millennium Ecosystem Assessment (2005). Ecosystems and Human Well-being: Biodiversity Synthesis. Washington, DC: World Resources Institute.

Moss, R. H., Edmonds, J. A., Hibbard, K. A., Manning, M. R., Rose, S. K., van Vuuren, D. P., Wilbanks, T. J. (2010). The next generation of scenarios for climate change research and assessment. Nature, 463(7282), 747-756. http://dx.doi.org/10.1038/ nature 08823

Parmesan, C. (1996). Climate and species' range. Nature, 382(6594), 765-766. http:// dx.doi.org/10.1038/382765a0

Parmesan, C. (2006). Ecological and Evolutionary Responses to Recent Climate Change. Annual Review of Ecology, Evolution, and Systematics, 37(1), 637-669. http://dx.doi. org/10.1146/annurev.ecolsys.37.091305.110100

Pauli, H., Gottfried, M., Dullinger, S., Abdaladze, O., Akhalkatsi, M., Alonso, J.L., Coldea, G., Dick, J., Erschbamer, B., Calzado, R.F., Ghosn, D., Holten, J.I., Kanka,R., Kazakis, G., Kollár, J., Larsson, P., Moiseev, P., Moiseev, D., Molau, U., Mesa,J.M., Nagy, L.,G, Pelino,G., Puşcaş, M., Rossi, G., Stanisci, A., Syverhuset, A.O., Theurillat, J.P., Tomaselli, M., Unterluggauer, P., Villar, L., Vittoz, P., Grabherr, G. (2012). Recent Plant Diversity Changes on Europe's Mountain Summits. Science, 336(6079), 353-355. http://dx.doi.org/10.1126/science.1219033

Pearson, R. G. (2007). Species Distribution Modeling for Conservation Educators and Practitioners. Lessons in Conservation, 3, 54-89.

Peterson, A. T. \& Lieberman, B. S. (2012). Species' Geographic Distributions through Time: Playing Catch-up with Changing Climates. Evolution: Education and Outreach, 5(4), 569-581. http://dx.doi.org/10.1007/s12052-012-0385-2

Phillips, S. J. (2010). A Brief Tutorial on Maxent. Lessons in Conservation, 3, 107-135. 
Phillips, S. J., Anderson, R. P. \& Schapire, R. E. (2006). Maximum entropy modeling of species geographic distributions. Ecological Modelling, 190(3-4), 231-259. http:// dx.doi.org/10.1016/j.ecolmodel.2005.03.026

Phillips, S. J., Dudík, M. \& Schapire, R. (2004). A maximum entropy approach to species distribution modeling. In Proceedings of the twenty-first Conference in Machine Learning. (pp. 1-8). Banff, Canada. http://dx.doi.org/10.1145/1015330.1015412

Pierce, J.R. (1980). An Introduction to Information Theory: Symbols, Signals and Noise. New York: Dover Publications, Inc.

Pimm, S. L., Jenkins, C. N., Abell, R., Brooks, T. M., Gittleman, J. L., Joppa, L. N., Sexton, J. O. (2014). The biodiversity of species and their rates of extinction, distribution, and protection. Science, 344(6187), 1246752. http://dx.doi.org/10.1126/science.1246752

Pliscoff, P. \& Fuentes-Castillo, T. (2011). Modelación de la distribución de especies y ecosistemas en el tiempo y en el espacio: una revisión de las nuevas herramientas y enfoques disponibles. Revista de Geografia Norte Grande, 48, 61-79. http://dx.doi. org/10.4067/S0718-34022011000100005

Prokopiuk, D. Cruz, G. Grados, N. Garro, O. \& Chiralt, A. (2000). Estudio comparativo entre frutos de Prosopis alba y Prosopis pallida. Piura: Facultad de Ingeniería, Universidad de Piura.

Riahi K., Rao, S., Krey, V., Cho, C., Chirkov, V., Fischer, G., Kindermann, G., Nakicenovic, N. \& Rafaj, P. (2011). A scenario of comparatively high greenhouse gas emissions. Climatic Change, 109: 33-57. http://dx.doi.org/10.1007/s10584-011-0149-y

Robinson, T. P., van Klinken, R. D. \& Metternicht, G. (2010). Comparison of alternative strategies for invasive species distribution modeling. Ecological Modelling, 221(19), 2261-2269. http://dx.doi.org/10.1016/j.ecolmodel.2010.04.018

Särkinen, T., Gonzales, P. \& Knapp, S. (2013). Distribution models and species discovery: the story of a new Solanum species from the Peruvian Andes. PhytoKeys, 20(31), 1-20. http://dx.doi.org/10.3897/phytokeys.31.6312

Seimon, T. A., Seimon, A., Daszak, P., Halloy, S. R. P., Schloegel, L. M., Aguilar, C. A., E. Simmons, J. (2007). Upward range extension of Andean anurans and chytridiomycosis to extreme elevations in response to tropical deglaciation. Global Change Biology, 13(1), 288-299. http://dx.doi.org/10.1111/j.1365-2486.2006.01278.x

Shannon, C. E. (1948). A mathematical theory of communication. The Bell System Technical Journal, 27(July 1928), 379-423. http://dx.doi.org/10.1002/j.1538-7305.1948. tb01338.x

Skolmen, R. (1990). Prosopis pallida H.B.K. En Burns, Russell M., Honkala, Barbara H. (Eds.), Silvics of North America: 2. Hardwoods. Agric. Handb. 654. Washington, DC: U.S. Department of Agriculture, Forest Service: 583-586.

Soberón, J. \& Peterson, A.T. (2004). Biodiversity informatics: Managing and applying primary biodiversity data. Philosophical Transactions of the Royal Society, B 35, 689698. http://dx.doi.org/10.1098/rstb.2003.1439 
Tovar, C., Arnillas, C. A., Cuesta, F. \& Buytaert, W. (2013). Diverging responses of tropical Andean biomes under future climate conditions. PloS One, 8(5), e63634.

Varela, S., Lobo, J. M. \& Hortal, J. (2011). Using species distribution models in paleobiogeography: A matter of data, predictors and concepts. Palaeogeography, Palaeoclimatology, Palaeoecology, 310(3-4), 451-463.

Walther, G., Post, E., Convey, P., Menzel, A., Parmesan, C., Beebee, T. J. C., Bairlein, F. (2002). Ecological responses to recent climate change. Nature, 416, 389-395.

Young, B.E. (2007). Distribución de las especies endémicas en la vertiente oriental de los Andes en Perú y Bolivia. Arlington: NatureServe.

Young, B.E., Franke, I. \& Hernandez, P. (2009). Using spatial models to predict areas of endemism and gaps in the protection of Andean slope birds. The Auk, 126(3), 554-565. 